\title{
Oncogenic role of microRNA-532-5p in human colorectal cancer via targeting of the 5'UTR of RUNX3
}

\author{
JIANTAO ZHANG ${ }^{1}$, WENLI ZHOU ${ }^{2}$, YANYAN LIU ${ }^{3}$, TAO LIU $^{1}$, CHENYAO LI $^{1}$ and LEI WANG ${ }^{1}$ \\ Departments of ${ }^{1}$ Colorectal and Anal Surgery, and ${ }^{2}$ Neonatology, First Affiliated Hospital of Jilin University, Changchun, \\ Jilin 130021; ${ }^{3}$ Department of Physiology, Harbin Medical University, Harbin, Heilongjiang 150081, P.R. China
}

Received October 9, 2016; Accepted August 17, 2017

DOI: $10.3892 / 01.2018 .8217$

\begin{abstract}
Previous studies have demonstrated that microRNAs (miRs) are involved in the carcinogenesis of colorectal cancer (CRC). To the best of our knowledge, the function and regulatory role of miR-532-5p in human CRC remains unknown. The aim of the present study was to determine the role and regulation of miR-532-5p in CRC. Using the reverse transcription-quantitative polymerase chain reaction, it was demonstrated that miR-532-5p was upregulated, whereas runt-related transcription factor 3 (RUNX3) was downregulated in CRC tissues. The upregulated miR-532-5p was associated with the downregulated RUNX3. Furthermore, the two biomarkers were associated with numerous clinicopathological characteristics of CRC, including tumor stage, lymph node involvement, differentiation, vessel invasion and tumor recurrence. The in vitro luciferase reporter assay demonstrated that transfection with miR-532-5p mimic markedly downregulated the RUNX3 mRNA and protein levels, via specific binding to the 5'-untranslated region of RUNX3 in human HT-29 CRC cells. In addition, an MTT assay and a colony formation assay demonstrated that miR-532-5p overexpression led to increased tumor cell viability and colony formation ability of HT-29 cells. In conclusion, the results of the present study indicate that miR-532-5p may function as an oncogenic miRNA by promoting cell growth in human CRC cells, and such promotion is associated with the targeted inhibition of RUNX3.
\end{abstract}

\section{Introduction}

Colorectal cancer (CRC), also known as colon cancer, rectal cancer or bowel cancer, is the third most common type of cancer in males and the second most common

Correspondence to: Dr Lei Wang, Department of Colorectal and Anal Surgery, First Affiliated Hospital of Jilin University, 71 Xinmin Street, Changchun, Jilin 130021, P.R. China

E-mail: leiwang368@sina.com

Key words: microRNA-532-5p, human colorectal cancer, runt-related transcription factor 3 in females worldwide in 2015 (1). In total 1,200,000 individuals develop CRC worldwide and 600,000 patients succumbed to the disease annually in 2014 (2). In total $>50 \%$ of the western population develops a colorectal tumor by the age of 70 years and for $\sim 10 \%$ of these individuals the tumors become malignant (3). Treatment approaches for patients with CRC have undergone marked changes in the last decade yet, despite the decline in mortality rates, CRC remains the third leading cause of cancer-associated mortality worldwide $(4,5)$. Previous research has identified the potential exploitation of microRNAs (miRNAs) as CRC biomarkers and demonstrated that aberrantly expressed miRNAs serve a role in CRC (4). Furthermore, it has been demonstrated previously that miRNAs may be involved in carcinogenesis (6).

miRNAs are a class of non-coding small single-stranded RNAs of between 18 and 25 nucleotides in length, which regulate gene expression by binding to specific sites at the 3' untranslated region (UTR) of target mRNAs. miRNAs are involved in numerous cellular and pathological processes including regulating cell proliferation, differentiation and apoptosis. MiRNAs also participate in tumor initiation and the progression of several human malignancies including CRC (7). It is becoming clear that miRNA expression is altered in numerous types of cancer, associates with tumor cell proliferation and may serve an oncogenic role in the cellular processes of cervical cancer (8-10).

miRNA-532-5p is located at human chromosome Xp11.23 and mature miRNA-532-5p consists of 22 nucleotides, with its sequence conserved between numerous species, indicating its importance (11). miR-532-5p is a regulatory factor of runt-related transcription factor 3 (RUNX3) expression and $R U N X 3$ is a known tumor suppressor gene of several types of carcinoma (12). Furthermore, miR-532-5p and RUNX3 are defined as cancer markers and therapeutic targets for cancer diagnosis, prognosis and treatment (13). To the best of our knowledge, the functional and regulatory role of miR-532-5p in human CRC remains unknown.

The present study was undertaken to reveal the regulatory role of miR-532-5p in CRC proliferation and to identify its association with the tumor suppressor gene $R U N X 3$. The results of the present study suggest that miR-532-5p promotes tumor cell growth via direct targeting of the 5'UTR of RUNX3 and functions in an oncogenic role in human CRC. 
Table I. Association of miRNA-532-5p level with clinicopathological characteristics of colorectal cancer.

\begin{tabular}{|c|c|c|c|}
\hline \multirow[b]{2}{*}{ Characteristic } & \multicolumn{3}{|c|}{$\operatorname{miR}-532-5 p$} \\
\hline & $\mathrm{n}$ & $\begin{array}{l}\text { Mean } \pm \text { standard } \\
\text { error of the mean }\end{array}$ & P-value \\
\hline Sex & & & 0.1711 \\
\hline Male & 35 & $1.823 \pm 0.1273$ & \\
\hline Female & 28 & $1.586 \pm 0.1140$ & \\
\hline Age, years & & & 0.6342 \\
\hline$\leq 55$ & 30 & $1.735 \pm 0.1284$ & \\
\hline$>55$ & 33 & $1.652 \pm 0.1158$ & \\
\hline Location & & & 0.1964 \\
\hline Colon & 36 & $1.788 \pm 0.1239$ & \\
\hline Rectum & 27 & $1.563 \pm 0.1098$ & \\
\hline T stage & & & $0.0125^{\mathrm{a}}$ \\
\hline $\mathrm{T} 1+\mathrm{T} 2$ & 19 & $1.371 \pm 0.1022$ & \\
\hline $\mathrm{T} 3+\mathrm{T} 4$ & 44 & $1.830 \pm 0.1083$ & \\
\hline $\mathrm{N}$ stage & & & $0.0145^{\mathrm{a}}$ \\
\hline$\leq \mathrm{N} 1$ & 21 & $1.399 \pm 0.1183$ & \\
\hline$>\mathrm{N} 1$ & 42 & $1.838 \pm 0.1078$ & \\
\hline Differentiation & & & 0.0818 \\
\hline High & 24 & $1.502 \pm 0.1463$ & \\
\hline Low & 39 & $1.808 \pm 0.1019$ & \\
\hline Vessel invasion & & & 0.1803 \\
\hline Positive & 66 & $1.366 \pm 0.1667$ & \\
\hline Negative & 7 & $1.732 \pm 0.0929$ & \\
\hline
\end{tabular}

${ }^{\mathrm{a}} \mathrm{P}<0.05$. $\mathrm{T}$ stage, tumor stage; $\mathrm{N}$ stage, lymph node involvement; miR, microRNA.

\section{Materials and methods}

CRC specimens, cell culture and reagents. The present study included 63 patients with CRC, who were registered at the Department of Colorectal and Anal Surgery of The First Affiliated Hospital of Jilin University (Changchun, China). The use of CRC samples was approved by the institutional review board of The First Affiliated Hospital of Jilin University according to the guidelines of the Dutch Federation of Medical Research Associations (14). Written informed consent was obtained from all 63 patients for the use of their CRC samples for research purposes. A total of 63 CRC tissues and 63 peri-CRC tissues $(1 \mathrm{~cm}$ away from the edge of tumors) were collected during eradication surgery from the patients with CRC who were registered in The First Affiliated Hospital of Jilin University between August 12th 2012 and October 24th 2014. No patients were subject to any radical resection or chemotherapy prior to surgery. Clinicopathological characteristics including age, sex, location, stage, differentiation and vessel invasion are presented in Table I.

The human HT-29CRC cell line was purchased from the American Type Culture Collection (Manassas, VA, USA).
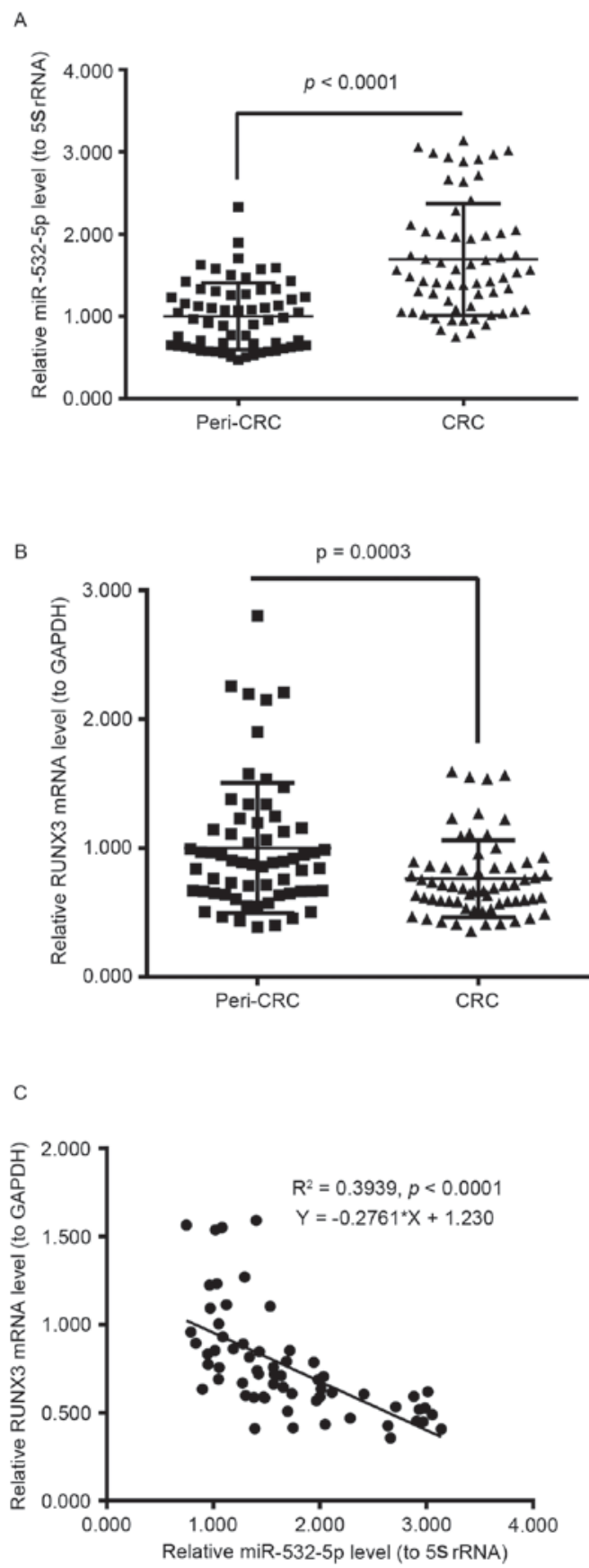

Figure 1. Increased miR-532-5p and decreased RUNX3 levels in human CRC tissues. (A) Relative miR-532-5p level to 5S rRNA in human CRC tissues $(n=63)$, with peri-tumor tissues $(n=63)$ as a control. (B) Relative mRNA level of RUNX3 to GAPDH in CRC $(n=63)$ or in peri-tumor tissues $(n=63)$. (C) Correlation of the increased miR-532-5p level with the decreased RUNX3 level in CRC tissues. miR, microRNA; RUNX3, runt-related transcription factor 3; CRC, colorectal cancer.

Cells were cultured in McCoy's 5a medium (Invitrogen; Thermo Fisher Scientific, Inc., Waltham, MA, USA), which was supplemented with $10 \%$ fetal bovine serum (FBS; GE Healthcare Life Sciences, Logan, UT, USA) and incubated at $37^{\circ} \mathrm{C}$ with $5 \% \mathrm{CO}_{2}$. For the miR-532-5p manipulation, 20 or $40 \mathrm{nM}$ miR-532-5p mimic or control miRNA (Ctrl miRNA; Merck KGaA, Darmstadt, Germany) were transfected with Lipofectamine RNAiMax (Invitrogen; Thermo Fisher Scientific, Inc.) into HT-29 cells at $85 \%$ confluence. 
A

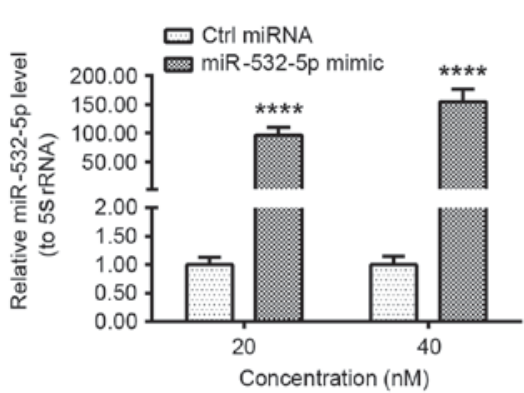

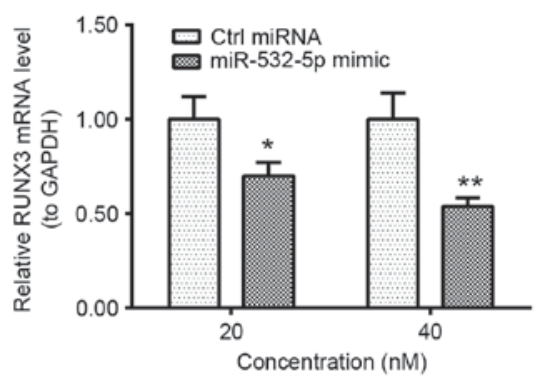

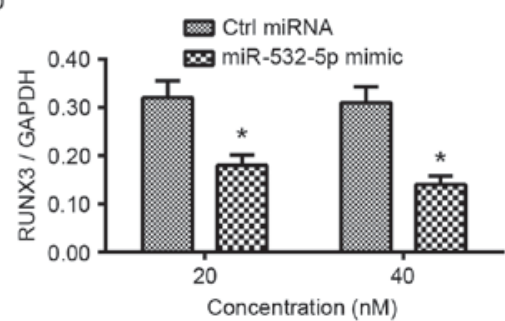

Figure 2. miR-532-5p downregulates RUNX3 expression in HT-29 CRC cells. HT-29 cells were transfected with 20 or $40 \mathrm{nM}$ miR-532-5p mimic or Ctrl miRNA for $12 \mathrm{~h}$. The relative miR-532-5p level to 5S rRNA (A) and the relative mRNA level of RUNX3 to GAPDH (B) were examined. (C) Western blot analysis of the RUNX3 protein level in the miR-532-5p mimic- or the Ctrl miRNA-transfected (for 24 h) HT-29 cells. (D) Expression levels of RUNX3 relative to GAPDH expression levels in HT-29 cells transfected with 20 or $40 \mathrm{nM}$ miR-532-5p mimic and Ctrl miRNA. Results are the mean \pm standard error of the mean from three independent experiments. ${ }^{*} \mathrm{P}<0.05 ;{ }^{* *} \mathrm{P}<0.01 ;{ }^{* * * * *} \mathrm{P}<0.0001$. miR, microRNA; RUNX3, runt-related transcription factor 3; CRC, colorectal cancer; Ctrl, control.

Preparation and quantitative analysis of $m R N A$ and miRNA samples. mRNA and miRNA samples from CRC tissues or from HT-29 cells were prepared respectively with the Recover All Total Nucleic Acid Isolation kit (Ambion; Thermo Fisher Scientific, Inc.) or with the mirPremier ${ }^{\mathrm{TM}}$ microRNA Isolation kit (Merck KGaA, Darmstadt, Germany), according to the manufacturer's protocol. For the quantitative analysis of miR-532-5p, the reverse transcription-quantitative polymerase chain reaction (RT-qPCR) was performed with the mirVana qRT-PCR miRNA Detection kit (Invitrogen; Thermo Fisher Scientific, Inc.) for each miRNA sample, with 5S rRNA as internal control. For the quantitative analysis of mRNA levels of $R U N X 3$, RT-qPCR was performed with the QuantiTect SYBR Green PCR kit (Qiagen GmbH, Hilden, Germany), with GAPDH as an internal control. The ${ }^{\Delta \Delta \mathrm{Cq}}$ method was used for relative quantification (15).

Western blot analysis for RUNX3. Cellular protein in HT-29 cells was prepared using a NE-PER Nuclear and Cytoplasmic Extraction Reagents kit (Pierce; Thermo Fisher Scientific, Inc.) and was treated with a Protease Inhibitor Cocktail (catalog no. ab65621; Abcam, Cambridge, MA, USA). The protein level of RUNX3 was evaluated using western blot analysis. In brief, protein samples were separated by SDS-PAGE (11\% gel) and transferred onto a hydrophobic polyvinylidene difluoride membrane. The RUNX3 band on the membrane was detected using the enhanced chemiluminescence detection system (GE Healthcare, Chicago, IL, USA), following the first incubation with rabbit anti-human $R U N X 3$ antibody (1:300; cat. no. ab49117; Abcam) or GAPDH (1:1,000; cat. no. ab9485; Abcam) at $4^{\circ} \mathrm{C}$ for $8 \mathrm{~h}$ and the second incubation with the horseradish peroxidase-conjugated anti-rabbit immunoglobulin $\mathrm{G}$ antibody (1:2,000; cat. no. A16104SAMPLE; Thermo
Fisher Scientific, Inc.) at room temperature for $1 \mathrm{~h}$. Prior to each incubation, membranes were washed four times in PBS.

Luciferase activity assay. miR-523-5p-targeted and control sequences were inserted into the 5'UTRs of the cytomegalovirus promoter of the luciferase reporter plasmid. For the luciferase reporter assay, $85 \%$ confluent $293 \mathrm{~T}$ cells, which were isolated from human embryonic kidneys and transformed with large $\mathrm{T}$ antigen (American Type Culture Collection, Rockville, MD, USA), were transfected for $6 \mathrm{~h}$ at $37^{\circ} \mathrm{C}$ with 20 or $40 \mathrm{nM}$ miRNA-523-5p or Ctrl miRNA. Medium was replaced with fresh Dulbecco's modified Eagle's medium containing 2\% FBS for a $24 \mathrm{~h}$ incubation at $37^{\circ} \mathrm{C}$. Cells were then harvested with a cell scraper (Corning Incorporated, Corning, NY, USA) for luciferase analysis. Luciferase activity was detected using a Rapid Detection of Firefly Luciferase Activity kit (cat. no. E1500; Promega Corporation, Madison, WI, USA).

Cell proliferation and colony formation assay. To investigate the regulatory action of miRNA-523-5p on the proliferation of CRC cells, a Cell Counting Kit-8 (CCK-8) assay (Dojindo Molecular Technologies, Inc., Kumamoto, Japan) and colony forming assay were performed for the HT-29 cells with miRNA-523-5p upregulation. HT-29 cells at $85 \%$ confluence were transfected with 0 or $40 \mathrm{nM}$ miRNA-523-5p mimic or Ctrl miRNA and incubated at $37^{\circ} \mathrm{C}$ for another $0,12,24$ and $48 \mathrm{~h}$. Cells were then incubated in CCK-8 reagent (Dojindo Molecular Technologies, Inc.). The $450 \mathrm{~nm}$ absorbance of each cell well was detected after the occurrence of visual color. For the cell colony formation assay, 100 HT-29 cells were incubated in 12-well plates and then transfected with 0 or $40 \mathrm{nM}$ miR-523-5p mimic or Ctrl miRNA for $72 \mathrm{~h}$ of incubation. Colonies were stained with $0.002 \%$ crystal violet 
Table II. Association of RUNX3 mRNA level with clinicopathological characteristics of colorectal cancer.

\begin{tabular}{|c|c|c|c|}
\hline \multirow[b]{2}{*}{ Characteristic } & \multicolumn{3}{|c|}{ Relative RUNX3 } \\
\hline & $\mathrm{n}$ & $\begin{array}{l}\text { Mean } \pm \text { standard } \\
\text { error of the mean }\end{array}$ & P-value \\
\hline Sex & & & 0.0623 \\
\hline Male & 35 & $0.8251 \pm 0.0553$ & \\
\hline Female & 28 & $0.6842 \pm 0.0458$ & \\
\hline Age, years & & & 0.3925 \\
\hline$\leq 55$ & 30 & $0.7284 \pm 0.0492$ & \\
\hline$>55$ & 33 & $0.7935 \pm 0.0564$ & \\
\hline Location & & & 0.4270 \\
\hline Colon & 36 & $0.7887 \pm 0.0550$ & \\
\hline Rectum & 27 & $0.7277 \pm 0.0488$ & \\
\hline T stage & & & $0.0355^{\mathrm{a}}$ \\
\hline $\mathrm{T} 1+\mathrm{T} 2$ & 19 & $0.8822 \pm 0.0838$ & \\
\hline $\mathrm{T} 3+\mathrm{T} 4$ & 44 & $0.7108 \pm 0.0381$ & \\
\hline $\mathrm{N}$ stage & & & 0.0592 \\
\hline$\leq \mathrm{N} 1$ & 21 & $0.8718 \pm 0.0591$ & \\
\hline$>\mathrm{N} 1$ & 42 & $0.7079 \pm 0.0477$ & \\
\hline Differentiation & & & $0.0079^{a}$ \\
\hline High & 24 & $0.8878 \pm 0.0681$ & \\
\hline Low & 39 & $0.6854 \pm 0.0399$ & \\
\hline Vessel invasion & & & $0.0359^{\mathrm{a}}$ \\
\hline Positive & 66 & $0.7348 \pm 0.0358$ & \\
\hline Negative & 7 & $0.9846 \pm 0.1695$ & \\
\hline Tumor recurrence & & & 0.0613 \\
\hline Positive & 43 & $0.7125 \pm 0.0461$ & \\
\hline Negative & 20 & $0.8700 \pm 0.0665$ & \\
\hline
\end{tabular}

${ }^{\text {a }} \mathrm{P}<0.05$. RUNX3, runt-related transcription factor 3; $\mathrm{T}$ stage, tumor stage; N stage, lymph node involvement.

(Sigma-Aldrich; Merck KGaA, Darmstadt, Germany) and were counted using the naked eye.

Statistical analysis. SPSS software (version 17.0; SPSS Inc., Chicago, IL, USA) was used for statistical analysis. Data are presented as the mean \pm standard error of the mean. Student's t-test was performed to evaluate the difference between two groups. Multiple comparisons between the groups were performed using the Student-Newman-Keuls method. Spearman's rank correlation coefficient was used to assess correlation between variables. $\mathrm{P}<0.05$ was considered to indicate a statistically significant difference.

\section{Results}

miR-532-5p and RUNX3 mRNA levels are increased and decreased, respectively, in human CRC tissues compared with peri-CRC tissues. To investigate miR-532-5p and RUNX3 mRNA levels in human CRC tissues, RT-qPCR was performed. The relative miR-532-5p levels to 5S rRNA in 63 human CRC tissues were significantly increased compared
A

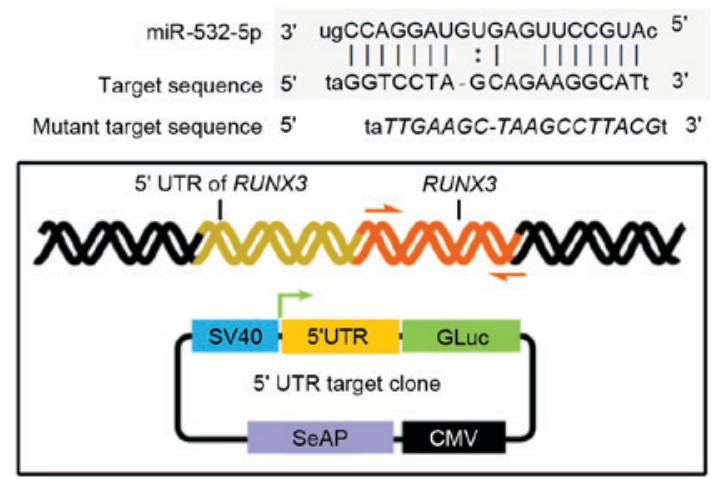

B

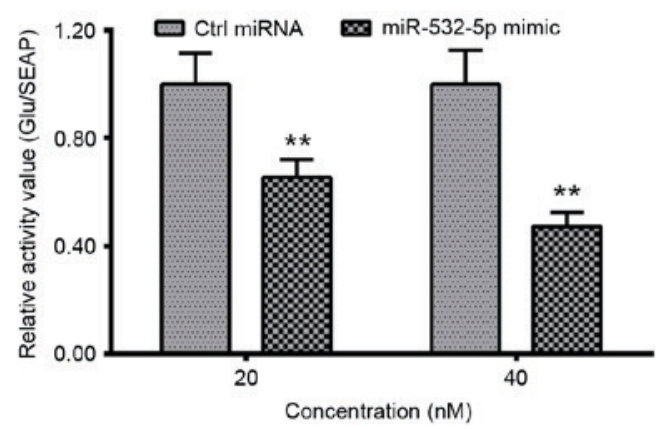

c

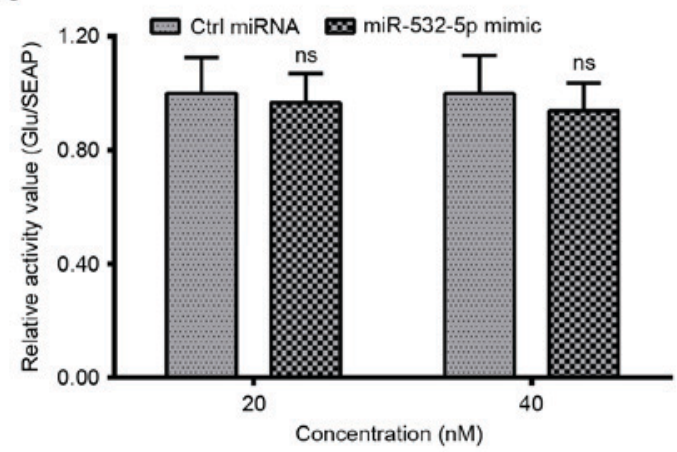

Figure 3. Targeting inhibition by miR-532-5p on the expression of RUNX3, with the luciferase reporting assay in HT-29 cells. (A) Alignment of Homo sapiens miR-532-5p with the target sequences within the 5'UTR of H. sapiens RUNX3 and a schematic diagram of the luciferase reporter with the 5'UTR of RUNX3 (wild-type or mutant 5'UTR of RUNX3). Relative luciferase activity of the reporter with the (B) $5^{\prime}$ UTR of RUNX3 or (C) mutant 5'UTR of RUNX3 in the HT-29 cells, following transfection with miR-532-5p mimic or with Ctrl miRNA. Experiments were performed in triplicate. ${ }^{* *} \mathrm{P}<0.01$, ns, not significant. SV40, simian virus 40; GLuc, Gaussia luciferase; SeAP, secreted embryonic alkaline phosphatase; CMV, cytomegalovirus; miR, microRNA; RUNX3, runt-related transcription factor 3; Ctrl, control; UTR, untranslated region.

with 63 peri-CRC tissues $(\mathrm{P}<0.0001$; Fig. $1 \mathrm{~A})$; and the relative RUNX3 mRNA levels in 63 human CRC tissues compared with 63 peri-CRC tissues were significantly decreased ( $\mathrm{P}=0.0003$; Fig. 1B). Furthermore, the scatter plot of the relative level association between miR-532-5p and RUNX3 mRNA from the $63 \mathrm{CRC}$ tissue samples revealed a negative association via Spearman's correlation $\left(\mathrm{R}^{2}=0.3939\right.$; $\mathrm{P}<0.0001$; Fig. 1C). These results demonstrated that the expression level of miR-532-5p was increased and the 


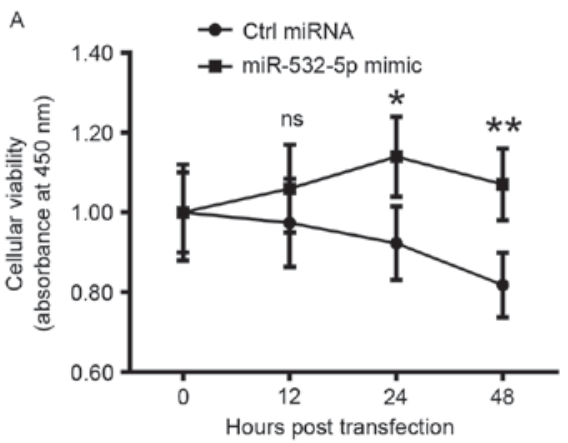

B

Blank

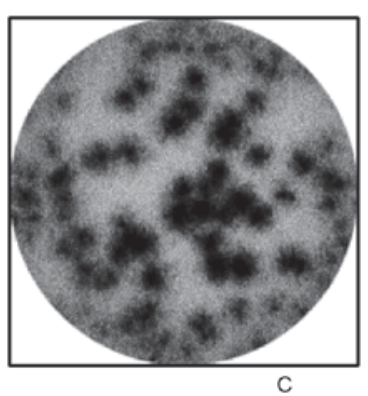

Ctrl miRNA
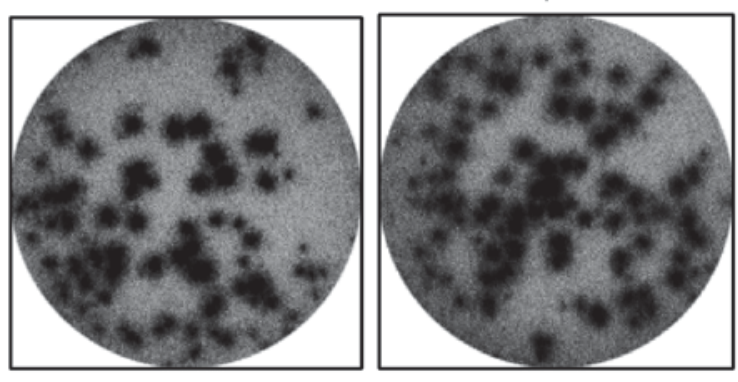

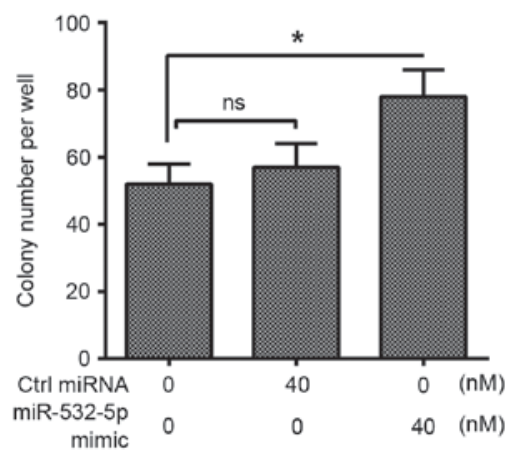

Figure 4. miR-532-5p promotes the proliferation and the colony formation of HT-29 cells. (A) Time-dependent promotion of the relative cellular proliferation of HT-29 cells by miRNA-532-5p mimic. (B) Morphology and (C) counting of the HT-29 cell colonies, which were transfected with 0 or $40 \mathrm{nM}$ miR-532-5p mimic or Ctrl miRNA, for $48 \mathrm{~h}$. The experiments were performed in triplicate. ${ }^{*} \mathrm{P}<0.05$; ${ }^{* *} \mathrm{P}<0.01$; ns, not significant. miR, microRNA; Ctrl miRNA, control miRNA.

expression level of RUNX3 mRNA was decreased in human CRC tissues.

RUNX3 mRNA and protein levels are associated inversely with miR-532-5p levels in HT-29 CRC cell lines. To investigate whether transfected miR-532-5p mimic affects RUNX3 levels, HT-29 cells were transfected with 20 or 40 nM miR-532-5p mimic or Ctrl miRNA for $12 \mathrm{~h}$. The relative miR-532-5p and RUNX3 mRNA levels were examined using RT-qPCR. Significantly increased levels of miR-532-5p were observed in 20 or $40 \mathrm{nM}$ miR-532-5p mimic-transfected HT-29 cells (Fig. 2A). The level of RUNX3 mRNA was significantly decreased in the transfected cell lines (Fig. 2B). To determine whether miR-532-5p had an effect on RUNX3 protein levels, western blot analysis was used. There was a decrease in RUNX3 protein quantities in 20 or $40 \mathrm{nM}$ miR-532-5p mimic-transfected cells (Fig. 2C). Quantification and statistical analysis of expression levels determined the decrease to be significant (Fig. 2D). These results demonstrated that miR-532-5p downregulated the mRNA and protein levels of RUNX3 in HT-29 CRC cell lines.
Targeting inhibition on RUNX3 expression by miR-532-5p. We hypothesize that RUNX3 may be a target of miR-532-5p. Homo sapiens miR-532-5p aligns with the target sequences with in the 5'UTR of $H$. sapiens RUNX3 (Fig. 3A). The luciferase reporter assay revealed a significant decrease in the relative activity value of the 5'UTR of RUNX3 in HT-29 cells following transfection with miR-532-5p mimic when compared with those transfected with Ctrl miRNA. The mutant 5'UTR of $R U N X 3$ group exhibited no significant difference between the relative activity values of cells transfected with miR-532-5p and cells transfected with Ctrl miRNA (Fig. 3C). These data suggest that the 5' UTR of RUNX3 is a functional target site for miR-532-5p in HT-29 CRC cells.

miR-532-5p promotes the growth of HT-29 CRC cells. miR-532-5p is upregulated in CRC, therefore it may serve a role in the proliferation of CRC cells. An MTT assay and colony formation assay were performed. The relative cellular viability was significantly increased in cells transfected with miR-532-5p mimic compared with Ctrl miRNA from 
$24 \mathrm{~h}$ after transfection onwards (Fig. 4A). Additionally, the colony formation assay analysis revealed that following transfection with miR-532-5p and Ctrl miRNA for $48 \mathrm{~h}$, the visible cultures of cells transfected with miR-532-5p covered more of the plate compared with those transfected with Ctrl miRNA (Fig. 4B). Quantification of the colony counts of the HT-29 cells also identified significantly upregulated quantities inmiR-532-5p-transfected cells (Fig. 4C). These results suggest that miR-532-5p may serve an oncogenic role in the proliferation and colony formation of CRC cells.

\section{Discussion}

miRNAs are small non-coding RNA molecules, involved in numerous cellular processes. There have been $>200$ mammalian miRNAs identified to date, with evidence suggesting that aberrantly expressed miRNAs are associated with a variety of diseases and serve roles in cell growth and apoptosis, including CRC (4,16-18). RUNX3 has been demonstrated to be a tumor suppressor and exhibits anti-cancer activity (19). Previous studies have demonstrated that aberrantly expressed miR-532-5p promoted cell growth in vitro (11) and regulated RUNX3 expression (12). However, little is known about the underlying molecular mechanism of miR-532-5p in CRC.

In the present study, aberrant expression of miR-532-5p and $R U N X 3$, the association between the two biomarkers, the target of miR-532-5p in CRC tissues and influence of miR-532-5p on the HT-29 cell viability and colony formation were investigated. Using RT-qPCR, it was identified that miR-532-5p was upregulated in CRC tissue; however, the RUNX3 miRNA level was downregulated in CRC tissue. miR-532-5p (Table I) and RUNX3 (Table II) were associated with several clinicopathological characteristics of CRC, including tumor stage, lymph node involvement, differentiation, vessel invasion and tumor recurrence.

Furthermore, it was demonstrated that both the mRNA and protein level of RUNX3 were significantly downregulated in miR-532-5p mimic-transfected HT-29 cells. Therefore, as previously reported, miR-532-5p is a regulatory factor of RUNX3 expression (20). The specific target site of miR-532-5p was also investigated in the present study. The 5'UTR of RUNX3 was demonstrated to be a functional target site for miR-532-5p in HT-29 cells using an in vitro luciferase reporting assay. Additionally, tumor cell viability and colony formation ability were promoted in overexpressed miR-532-5p HT-29 cells. Taken together, the results of the present study demonstrate that the tumor suppressor RUNX3 is negatively regulated by miR-532-5p at the 5'UTR of RUNX3. The present study is, to the best of our knowledge, the first to investigate the underlying molecular mechanism of miR-532-5p in CRC cells.

To conclude, it was revealed that miR-532-5p was positively regulated in CRC cells; by contrast, RUNX3 mRNA levels were negatively regulated in CRC cells. Furthermore, it was demonstrated that miR-532-5p significantly downregulated mRNA and protein levels of RUNX3. It was also identified that miR-532-5p may serve an oncogenic role in CRC proliferation and colony formation via targeting of the specific site at the 5'UTR of RUNX3. The results of the present study may provide insight into novel therapeutic approaches towards CRC.

\section{References}

1. Ferlay J, Soerjomataram I, Dikshit R, Eser S, Mathers C, Rebelo M, Parkin DM, Forman D and Bray F: Cancer incidence and mortality worldwide: Sources, methods and major patterns in GLOBOCAN 2012. Int J Cancer 136: E359-E386, 2015.

2. Brenner H, Kloor M and Pox CP: Colorectal cancer: Lancet 383: 1490-1502, 2014.

3. Kinzler KW and Vogelstein B: Lessons from hereditary colorectal cancer. Cell 87: 159-170, 1996.

4. Okugawa Y, Toiyama Y and Goel A: An update on microRNAs as colorectal cancer biomarkers: Where are we and what's next? Expert Rev Mol Diagn 14: 999-1021, 2014.

5. Moriarity A, O'Sullivan J, Kennedy J, Mehigan B and McCormick P: Current targeted therapies in the treatment of advanced colorectal cancer: A review. Ther Adv Med Oncol 8: 276-293, 2016.

6. Bandres E, Agirre X, Bitarte N, Ramirez N, Zarate R, Roman-Gomez J, Prosper F and Garcia-Foncillas J: Epigenetic regulation of microRNA expression in colorectal cancer. Int $\mathrm{J}$ Cancer 125: 2737-2743, 2009.

7. Bovell L, Putcha BDK, Devadasan D, Bae S, Grizzle WE and Manne U: Abstract 1944: Evaluation of the prognostic value of miRNA-181b and its target identification and validation in colorectal cancers. Cancer Res 73 (8 Suppl): 1944, 2013.

8. Yao Q, Xu H, Zhang QQ, Zhou H and Qu LH: MicroRNA-21 promotes cell proliferation and down-regulates the expression of programmed cell death 4 (PDCD4) in HeLa cervical carcinoma cells. Biochem Biophys Res Commun 388: 539-542, 2009.

9. Chan JA, Krichevsky AM and Kosik KS: MicroRNA-21 is an antiapoptotic factor in human glioblastoma cells. Cancer Res 65: 6029-6033, 2005.

10. Motoyama K, Inoue H, Takatsuno Y, Tanaka F, Mimori K, Uetake H, Sugihara K and Mori M: Over- and under-expressed microRNAs in human colorectal cancer. Int J Oncol 34: 1069-1075, 2009.

11. Xu X, Zhang Y, Liu Z, Zhang X and Jia J: miRNA-532-5p functions as an oncogenic microRNA in human gastric cancer by directly targeting RUNX3. J Cell Mol Med 20:95-103, 2016.

12. Kitago M, Martinez SR, Nakamura T, Sim MS and Hoon DS: Regulation of RUNX3 tumor suppressor gene expression in cutaneous melanoma. Clin Cancer Res 15: 2988-2994, 2009.

13. Hoon DSB and Kitago M: Use of runx3 and mir-532-5p as cancer markers and therapeutic targets. US, 2009.

14. Kloth JN, Kenter GG, Spijker HS, Uljee S, Corver WE, Jordanova ES, Fleuren GJ and Gorter A: Expression of Smad2 and Smad4 in cervical cancer: Absent nuclear Smad4 expression correlates with poor survival. Mod Pathol 21: 866-875, 2008.

15. Livak KJ and Schmittgen TD: Analysis of relative gene expression data using real-time quantitative PCR and the 2(-Delta Delta C(T)) method. Methods 25: 402-408, 2001.

16. Chen X, Guo X, Zhang H, Xiang Y, Chen J, Yin Y, Cai X, Wang K, Wang G, Ba Y, et al: Role of miR-143 targeting KRAS in colorectal tumorigenesis. Oncogene 28: 1385-1392, 2009.

17. Cheng AM, Byrom MW, Shelton J and Ford LP: Antisense inhibition of human miRNAs and indications for an involvement of miRNA in cell growth and apoptosis. Nucleic Acids Res 33: 1290-1297, 2005.

18. Slaby O, Svoboda M, Fabian P, Smerdova T, Knoflickova D, Bednarikova M, Nenutil R and Vyzula R: Altered expression of miR-21, miR-31, miR-143 and miR-145 is related to clinicopathologic features of colorectal cancer. Oncology 72: 397-402, 2007.

19. Li QL, Ito K, Sakakura C, Fukamachi H, Inoue K, Chi XZ, Lee KY, Nomura S, Lee CW, Han SB, et al: Causal relationship between the loss of RUNX3 expression and gastric cancer. Cell 109: 113-124, 2002.

20. Asangani IA, Rasheed SA, Nikolova DA, Leupold JH, Colburn NH, Post S and Allgayer H: MicroRNA-21 (miR-21) post-transcriptionally downregulates tumor suppressor Pdcd4 and stimulates invasion, intravasation and metastasis in colorectal cancer. Oncogene 27: 2128-2136, 2008. 